\begin{tabular}{|l|c|c|c|r|}
\hline $\begin{array}{l}\text { Cuadernos de Investigación Geográfica } \\
\text { Geographical Research Letters }\end{array}$ & 2018 & N $^{\circ} 44(2)$ & pp. 675-695 & $\begin{array}{r}\text { ISSN 0211-6820 } \\
\text { eISSN 1697-9540 }\end{array}$ \\
\hline
\end{tabular}

\title{
TÉCNICAS DE RESTAURACIÓN DE SUELOS BASADAS EN EL USO DE RESIDUOS ORGÁNICOS: SEIS AÑOS DE BENEFICIOS SOBRE LAS PROPIEDADES DE UN SUELO FORESTAL
}

\author{
P. HUESO-GONZÁLEZ ${ }^{1 *}$, J.M. MARTÍNEZ-MURILLO ${ }^{1}$, J.D. RUIZ-SINOGA ${ }^{1}$ \\ ${ }^{1}$ Departamento de Geografía, Universidad de Málaga. Instituto de Geomorfología y Suelos \\ (IGSUMA), Andalucía Tech. Campus de Teatinos s/n, 29071 Málaga, España.
}

RESUMEN: Restaurar la vegetación nativa es una de las maneras más efectivas de recuperar la calidad de los suelos forestales. Después de la repoblación, durante el enraizamiento del plantón, los beneficios de la vegetación sobre el suelo aún no se manifiestan y los suelos siguen siendo altamente vulnerables a la erosión y pérdida de carbono orgánico. Los procesos de degradación podrían minimizarse mediante la implementación de enmiendas orgánicas. El objetivo es analizar los beneficios del uso de diferentes enmiendas orgánicas sobre algunas propiedades del suelo. Los tratamientos seleccionados fueron: (i) suelos reforestados y enmendados con un acolchado de restos de paja; (ii) suelos reforestados y enmendados con un acolchados de astillas de pino carrasco (Pinus halepensis Mill.); (iii) suelos reforestados y enmendados con lodos de depuradora; (iv) suelos reforestados y enmendados con estiércol de origen vacuno y; $(v)$ no enmendados. Todas las enmiendas se aplicaron a dosis de $10 \mathrm{Mg} \mathrm{ha}^{-1}$. Seis años después de su aplicación, únicamente la adición de enmiendas orgánicas, tipo acolchado de paja o poda, han supuesto un incremento significativo de los valores de carbono orgánico del suelo respecto a la reforestación bajo condiciones de suelo desnudo. Este aumento estaba directamente relacionado con el aumento de la actividad microbiológica que favorece el incremento de la estabilidad media de los agregados. Los tratamientos con lodos de depuradora o estiércol de origen vacuno no serían métodos útiles para la mejora de la calidad del suelo.

Restoration techniques applying organic amendments: six years of benefits on soil forest properties

ABSTRACT: Restoring the native vegetation is one of the most effective way to regenerate forest soil health. The seeding and plant establishment stages are critical; but during these stages the beneficial effects of the vegetation may not be apparent, and the soil is highly susceptible to erosion and depletion of soil quality. In the initial months after afforestation, vegetation cover establishment and soil quality could be better sustained if the soil was amended with an external extra source of organic matter. The objective of this study is to analyze the benefits 
of using different organic amendments on some soil properties. The soil treatment selected were: (i) afforestation with straw mulching treatment; (ii) afforestation with mulch with chipped branches of Aleppo Pine (Pinus halepensis Mill.); (iii) afforestation with cattle manure compost; (iv) sewage sludge and; (v) afforestation in unamended soils, control condition. The amendments were applied at the rate of $10 \mathrm{Mg} \mathrm{ha}^{-1}$. Six years after the amendment application, only the addition of straw and pine mulch have shown a significant increase in soil organic carbon regarding the afforestation under bare soil conditions. Besides, this increase was also directly related with the increase in microbiological activity and aggregate stability. On the other hand, the addition of sewage sludge or cattle manure is not an effective treatment to favor the edaphic structure regarding the afforestation under unamended soils.

Palabras clave: enmiendas orgánicas, reforestación, carbono orgánico, estabilidad estructural de agregados, deshidrogenasa.

Key words: organic amendments, afforestation, soil organic carbon, aggregate stability, dehydrogenase.

Recibido: 17 de septiembre de 2017 Aceptado: 17 de octubre de 2017

*Correspondencia: Paloma Hueso-González, Departamento de Geografía, Universidad de Málaga, Instituto de Geomorfología y Suelos (IGSUMA), Andalucía Tech. Campus de Teatinos s/n, 29071 Málaga, España.E-mail: phueso@uma.es

\section{Introducción}

Los suelos constituyen un recurso básico por lo que su "salud" debe de ser conservada en aras de mantener la productividad y diversidad de los ecosistemas terrestres (COM, 2002; Eaton et al., 2008). Así, juegan un papel fundamental en la provisión de alimentos, ayudan a mantener la calidad de aire y agua y son grandes proveedores de biodiversidad (Keesstra et al., 2016). Es por ello, que su protección y conservación cobra especial relevancia cuando hablamos de ecosistemas frágiles o ecológicamente sensibles (Martínez-Murillo et al., 2016). En las áreas semiáridas Mediterráneas, las actividades antrópicas (arado en pendientes, deforestación y sobrepastoreo) y la alternancia climática de largos periodos de sequía, que se intercalan con periodos cortos de precipitación intensa, han conllevado una intensa degradación de los suelos generando una pérdida de diversidad sistémica (Casals et al., 2000; Muñoz-Rojas et al., 2016; Martínez-Murillo et al., 2017a, 2017b). Concretamente, en los suelos forestales, esto es porque la dinámica del carbono orgánico del suelo (SOC) suele estar condicionada por aquellos factores relativos a la temperatura y humedad del suelo (RuizSinoga et al., 2009; Hueso-González et al., 2014; Bretón et al., 2016).

Restaurar la vegetación nativa es una de las maneras más efectivas de recuperar la salud de los suelos forestales (Hueso-González et al., 2015; Nadal-Romero et al., 2016). Sin embargo, después de una repoblación, durante los primeros estadios de la vegetación, 
siembra y establecimiento del plantón, los beneficios de la vegetación sobre el suelo aún no se hacen manifiestos y los suelos siguen siendo altamente vulnerables a la erosión y pérdida de carbono orgánico (Maestre et al., 2003; Hueso-González et al., 2016). En estos primeros años, se ha demostrado que los procesos de degradación podrían minimizarse mediante la implementación de técnicas encaminadas a la protección del suelo como son el uso de residuos orgánicos tanto animales como vegetales (Jordán et al., 2010; Bretón et al., 2016; Pal y Mahajan, 2017; Wahbi et al., 2017). En este sentido, el efecto de varias enmiendas orgánicas, incluidas lodos de depuradora, estiércol o acolchados orgánicos ha sido objeto de numerosos estudios (Guerrero et al., 2001; Ojeda et al., 2003; Tejada y González, 2003; Jordán et al., 2011; Jiménez et al., 2013; Iglesias et al., 2015; Dudás et al., 2016; Prosdocimi et al., 2016).

En términos generales, la adición de residuos orgánicos al suelo se fundamenta en el principio de que las enmiendas aumentan el contenido de materia orgánica debido a los procesos de descomposición de las mismas (Hueso-González et al., 2017). A largo plazo, se espera un aumento del porcentaje de agregados estables y el aumento el espacio poroso (Ojeda et al., 2015; Luna et al., 2016). A su vez, las implicaciones que estos cambios tendrían sobre la hidrología del sistema podrían suponer un aumento para los umbrales de escorrentía y el descenso de las tasas de erosión (Lavee et al., 1998). Así, lo describen Tejada y González (2003) para un suelo agrícola enmendado con dos compost orgánicos a las dosis de 3,5 y 7,0 $\mathrm{Mg} \mathrm{Ha}^{-1}$. En esta línea están también los resultados descritos por Flanagan et al. (1997a, 1997b), quienes afirman que tras la adición al suelo de un compost de ceniza vegetal los cambios producidos en las propiedades del suelo, incremento de materia orgánica y estabilidad estructural, aumentan significativamente las tasas de infiltración en el perfil (41,9 $\mathrm{mm} \mathrm{h}^{-1} \mathrm{vs} 32,2 \mathrm{~mm} \mathrm{~h}^{-1}$ para el control). Por otro lado, la aplicación de enmiendas orgánicas a los suelos podría suponer una alteración del pH y la conductividad eléctrica (CE) (Guang-Ming et al., 2006). Además, existen estudios específicos que demuestran que en sistemas agrícolas donde la vegetación aún no está establecida las enmiendas orgánicas pueden ser utilizadas como técnicas para proteger al suelo frente a las fuerzas de la lluvia y la escorrentía (Smets et al., 2008; Gholami et al., 2012; Prats et al., 2014). Un estudio llevado a cabo en condiciones agrícolas semiáridas demostraba la efectividad de los mulch para aumentar la rugosidad superficial y disminuir las pérdidas de suelo respecto a los suelos control (Jordán et al., 2010). De forma similar, otros estudios han demostrado que el tratamiento del suelo con polímeros hidro-absorbentes reduce el efecto splash por salpicadura y previene el sellado superficial (Hueso-González et al., 2015).

Todos los estudios hasta ahora mencionados demuestran el efecto positivo que el uso de enmiendas de diversa naturaleza tiene sobre las propiedades de los suelos agrícolas, especialmente aquellas directamente relacionadas con el incremento de SOC y mejora de la estructura edáfica. Sin embargo, el uso de las enmiendas y su aplicación en sistemas forestales repoblados aún no está bien descrito. Ante este nuevo paradigma, la estrategia de la Unión Europea, en materia de economía circular, está enfocada a utilizar de forma eficiente los recursos disponibles, optimizando los flujos de materiales, el consumo de energía y la gestión de los residuos. Por ello, en la Directiva Marco de Residuos (DMR, 2008) se establece la obligatoriedad de todos los Estados miembros de tomar medidas para fomentar la prevención, la reutilización de los productos y las actividades de preparación 
para la reutilización y el reciclado, garantizando el cumplimiento de una serie de objetivos claramente definidos de reducción, preparación para la reutilización, reciclado y valorización (Keesstra et al., 2018). Por ello, el objetivo de este estudio es analizar los beneficios del uso de diferentes enmiendas orgánicas sobre algunas propiedades del suelo y en los estadios iniciales de una repoblación forestal. Concretamente, seis años después de la reforestación, se ha evaluado el efecto de cuatro enmiendas orgánicas sobre los siguientes parámetros: (i) propiedades químicas: carbono orgánico del suelo (SOC); pH y conductividad eléctrica (CE); (ii) propiedades físicas: estabilidad estructural de los agregados del suelo (AGS); y (iii) propiedades microbiológicas: actividad enzimática de la deshidrogenasa en el suelo (DSH).

\section{Materiales y métodos}

\section{1. Área experimental}

El área experimental "El Pinarillo" se encuentra ubicada a 470 m.s.n.m., en la parte alta de un abanico aluvial formado por conglomerados calcáreos y dentro del Parque Natural Sierra Tejeda, Almijara y Alhama (sureste de España; Fig. 1; X: 424.240 m; Y: 4.073.098 m; UTM30N/ED50) (Hueso-González et al., 2014, 2015, 2016, 2017, 2018) . Para el diseño experimental se construyeron 10 parcelas experimentales simultáneas en tiempo. Se trata de parcelas experimentales cerradas de $2 \mathrm{~m}$ de ancho y $12 \mathrm{~m}$ de longitud, $24 \mathrm{~m}^{2}$ de superficie total, que se han orientado de forma paralela a la línea de máxima pendiente. Así, la pendiente registrada para las 10 parcelas se corresponde con un 7,5\% y la orientación es $\mathrm{N} 170^{\circ}$. Para garantizar la uniformidad de las parcelas, la cubierta vegetal que de forma natural existía en el área fue eliminada. Esto se hizo con el fin de evitar interferencias en los resultados. Finalmente, los restos vegetales procedentes del desbroce de la vegetación fueron retirados.

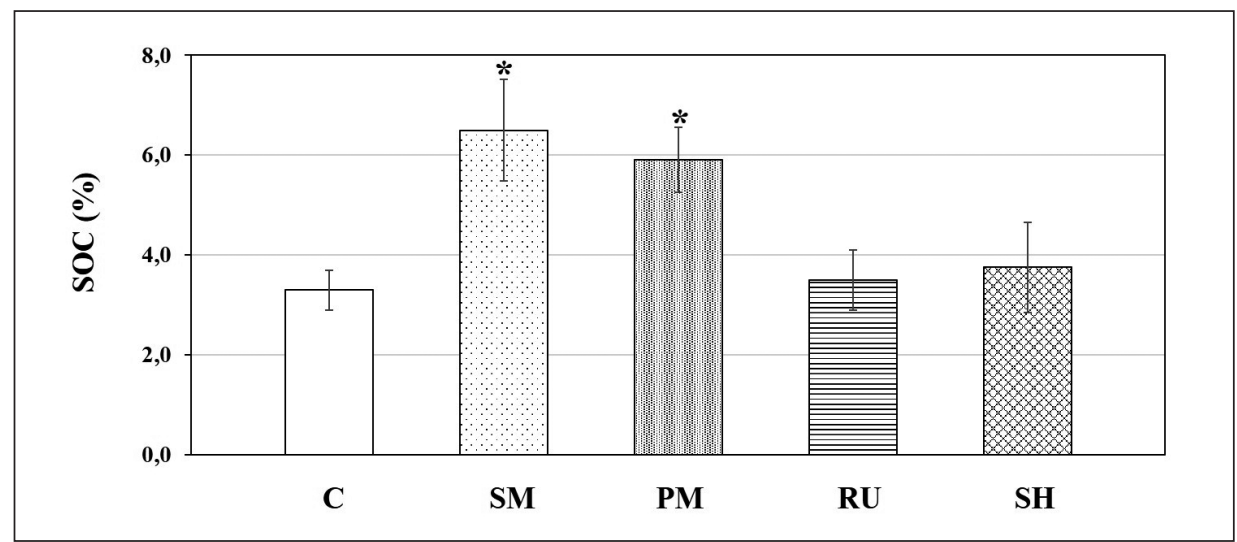

Figura 1. Localización del área experimental. Donde: a, suelos reforestados y no enmendados (control); $b$, suelos reforestados y enmendados con un acolchado de restos paja (10 Mg ha $\left.{ }^{-1}\right) ; c$, suelos reforestados y enmendados con un acolchados de astillas de pino (Pinus halepensis Mill.)

(10 $\left.\mathrm{Mg} \mathrm{ha}^{-1}\right) ; d$, suelos reforestados y enmendados con lodos de depuradora (10 $\left.\mathrm{Mg} \mathrm{ha}^{-1}\right) ; e$, suelos reforestados y enmendados con estiércol de origen vacuno $\left(10 \mathrm{Mg} \mathrm{ha}^{-1}\right)$. 


\subsection{Clima y Suelo}

El clima es seco-mediterráneo (temperatura media de $18^{\circ} \mathrm{C}$, precipitación media $589 \mathrm{~mm}$ año ${ }^{-1}$ ). La vegetación dominante consiste en un bosque de pino abierto (Pinus halepensis Mill.) acompañado del típico matorral mediterráneo. Las parcelas experimentales se encuentran ubicadas en la cara sur de una ladera con exposición solana y con uso agrícola hasta mediados de los años 1950. Además, la ladera se ha visto expuesta a los efectos del fuego en los veranos de 1975 y 1991.

Los suelos son del tipo Leptosoles líticos y eútricos con textura franco arenosa (arena $=60 \%$, limo $=32 \%$, arcilla $=8 \%)$ y presentan un elevado contenido en gravas (total de gravas $=56 \%$; gravas $>\mathrm{f} 10 \mathrm{~mm}=31 \%$; grava $\mathrm{f} 2 \mathrm{~mm}=10 \%$; grava $\mathrm{f} 1 \mathrm{~mm}=15 \%$ ). El perfil edáfico dominante es tipo $\mathrm{ACk} / \mathrm{Bw}$, resultado de una elevada fracturación en la roca madre y con contenido de carbono orgánico medio-bajo (LUCDEME, 1986). Las propiedades y características específicas de los suelos se encuentran recogidas en la Tabla 1 .

Tabla 1. Propiedades del suelo bajo condiciones naturales en el área experimental El Pinarillo. Octubre 2010. CIC: capacidad de intercambio catiónico; CT: Carbono total; NT; Nitrógeno total; C/N: ratio carbono/nitrógeno; EC: Conductividad eléctrica; SOC: contenido de carbono orgánico; SWC: Contenido de agua en el suelo.

\begin{tabular}{lll}
\hline Precipitación anual & $\left(\mathrm{mm} \mathrm{año}^{1}\right)$ & 589 \\
Régimen climático & & Seco \\
Especies dominantes & & Chamaerops humilis, Cistus albidus, \\
& & Rosmarinus officinalis
\end{tabular}

Propiedades del suelo

Humedad

$\begin{array}{rll}\text { Gravas } & (\%) & 56 \\ \text { Arena } & (\%) & 60 \\ \text { Limo } & (\%) & 32 \\ \text { Arcilla } & (\%) & 8 \\ \text { MAGSs } & (\%) & 34,1 \\ \text { mAGS } & (\%) & 64,2 \\ C I C & \text { meq } 100 \mathrm{~g}^{-1} & 120,4 \\ C T & (\%) & 12,5 \\ N T & (\%) & 0,2 \\ C / N & & 74 \\ \text { SOC } & (\%) & 2,9 \\ p H & & 8 \\ E C & \left(\mu \mathrm{S} \mathrm{cm}^{-1}\right) & 501 \\ & (\%) & 8,7\end{array}$

Conductividad hidráulica

\begin{tabular}{lll}
$-0,5 \mathrm{~cm}$ & $\left(\mathrm{~cm} \mathrm{~h}^{-1}\right)$ & 1,54 \\
$-2,0 \mathrm{~cm}$ & $\left(\mathrm{~cm} \mathrm{~h}^{-1}\right)$ & 0,56 \\
$-6,0 \mathrm{~cm}$ & $\left(\mathrm{~cm} \mathrm{~h}^{-1}\right)$ & 0,42 \\
Capacidad de campo SWC & $(\%)$ & 25,2 \\
Punto de marchitez SWC & $(\%)$ & 6,4 \\
Agua disponible & $(\%)$ & 18,8 \\
\hline
\end{tabular}




\subsection{Enmiendas}

En mayo de 2011, se añade al suelo, en 8 de las 10 parcelas, 4 tipos diferentes de enmiendas orgánicas a las dosis de $10 \mathrm{Mg} \mathrm{ha}^{-1}$. Las enmiendas seleccionadas fueron: (i) acolchado de restos de paja (SM); (ii) acolchados de astillas de pino carrasco (Pinus halepensis Mill.) (PM); (iii) lodos de depuradora (RU); y (iv) estiércol de origen vacuno $(\mathrm{SH})$. En este experimento, cada tratamiento ha sido replicado en dos parcelas experimentales y el patrón de repetición ha sido al azar.

\subsection{Reforestación}

En noviembre de 2011, seis meses después de la aplicación de las enmiendas, las 10 parcelas han sido reforestadas con el mismo número de plantas y patrón espacial de arbustos mediterráneos. Así, la vegetación se ha reforestado utilizando un patrón al tres bolillo con $0,5 \mathrm{~m}$ de distancia entre plantones. Durante el proceso de reforestación, el suelo se volteó hasta los $25 \mathrm{~cm}$ de profundidad desmantelando completamente la estructura del suelo. Las especies vegetales utilizadas para la reforestación han sido Lavadula stoechas Lam., L. dentatae L., L. multifida L., R. officinalis L. y T. capitatus $L$. En este estudio la condición control (C) la conforman 2 parcelas experimentales que fueron reforestadas pero no enmendadas.

\subsection{Muestreo, análisis de las propiedades del suelo y mediciones}

El suelo de las 10 parcelas se muestreó en la primavera del año 2016 (60 meses después de la adición de las enmiendas). La estrategia de muestreo para cada parcela consistió en la recolección de 4 muestras de suelo perturbadas tomadas de la superficie (0-10 cm de profundidad). Las propiedades del suelo analizadas fueron: i) salinidad del suelo (CE), que se midió en una suspensión de agua desionizada del suelo (5:1) utilizando un medidor de conductividad Crisol Micro CM 2200 (ISRIC, 2002); ii) acidez del suelo $(\mathrm{pH})$, que se midió en una suspensión de agua desionizada del suelo $(2,5: 1)$ usando un medidor de pH Crisol GLP 21; iii) carbono orgánico del suelo (SOC), que se determinó utilizando el método Walkley-Black de oxidación con dicromato y titulación posterior (FAO, 2006); iv) agregación del suelo (AGS), se basó en la medida de la estabilidad media estructural de los agregados $(\leq 2 \mathrm{~mm})$. El procedimiento utilizado fue por el método de Wet-Sieving (Kemper y Rosenau, 1986; Madari et al., 2005); y v) actividad enzimática para la deshidrogenasa (DHS), se basó en la determinación clorimétrica del producto liberado 2, 3, 5-trifenilformazan (TPF) a $485 \mathrm{~nm}$ tras la incubación a $37^{\circ}$ por $24 \mathrm{~h}$ de muestra de suelo con cloruro de 2, 3, 5-trifeniltetrazolio (CTT, Casida et al., 1964), usando como referencia el metanol. La curva de calibración se preparó en función de las concentraciones: 4, 12, 20 y $40 \mu \mathrm{g}$ de TPF $\mathrm{ml}^{-1}$. La absorbancia del blanco se restó con las absorbancias de cada una de las muestras, y a partir de la curva de calibración se calcularon las concentraciones de la misma en $\mu \mathrm{g}$ de TPF $\mathrm{ml}^{-1}$. La DHS, expresada en $\mu \mathrm{g}$ de TPF $\mathrm{g}^{-1}$ PS $24 \mathrm{~h}^{-1}$, se calculó mediante la fórmula:

$$
D H S=(M-B) * V F / P S
$$


Donde:

M: concentración de la muestra $\left(\mu \mathrm{g}\right.$ de TPF $\left.\mathrm{ml}^{-1}\right)$

B: concentración del blanco ( $\mu \mathrm{g}$ de TPF $\left.\mathrm{ml}^{-1}\right)$

VF: volumen final en el matraz aforado ( $\mu \mathrm{g}$ de TPF $\left.\mathrm{ml}^{-1}\right)$

PS: peso seco de la muestra (g)

\subsection{Tratamiento estadístico}

Las diferencias estadísticamente significativas se determinaron mediante el análisis de varianza (ANOVA). En todos los análisis el límite de significación seleccionado fue $p<0,05$. La asunción de homoscedasticidad se probó usando el test de Levene. En los casos de no homoscedasticidad (test de Levene, $p<0,05$ ), se utilizaron pruebas no paramétricas. Las diferencias medias entre los diferentes tratamientos experimentales del suelo se determinaron usando el test de Tukey o la prueba de Games-Howell. Finalmente, se correlacionaron los datos de actividad enzimática con las diferentes variables del análisis químico de suelos. Todos los análisis se han realizado con el software SPSS (versión 21) para Windows.

\section{Resultados}

\subsection{Propiedades químicas}

En la Figura 2, se observa el efecto de los cinco tratamientos sobre el SOC. En términos generales, el SOC en el conjunto $\mathrm{C}$ mantenía valores muy similares $(3,1 \pm$ $0,2 \%$ ) a los encontrados seis años antes bajo condiciones de suelo desnudo (SOC = 2,9\%; Tabla 1). Sin embargo, cuando los suelos se enmendaban, se observaban dos tendencias totalmente opuestas: i) aquellas enmiendas que, tras seis años de exposición, incrementaban el SOC respecto a $\mathrm{Cy}$; ii) aquellas enmiendas en las que los valores de SOC se mantenían similares a los encontrados conjunto control. En este sentido, los resultados del ANOVA muestran un aumento significativo del porcentaje de SOC únicamente para los tratamientos PM y SM ( $p \leq 0,05$; Fig. 2). Concretamente, el porcentaje de SOC medido fue de $6,5 \pm 0,2 \%$ y $5,9 \pm 0,6 \%$ para los conjuntos SM y PM, respectivamente, frente al 3,1 $\pm 0,2 \%$ medido en $\mathrm{C}$.

Las figuras 3 y 4 contienen los valores de $\mathrm{pH}$ y $\mathrm{CE}$ de los cinco tratamientos objeto de estudio. En la figura 3, se puede observar como, con independencia de la enmienda añadida al suelo, el pH del suelo no mostraba diferencias significativas respecto a $\mathrm{C}(p \geq 0,05)$. Así, los valores de $\mathrm{pH}$ encontrados fueron similares, con valores de $7,9 \pm 0,1 ; 8,0 \pm 0,0 ; 8,1 \pm 0,0 \mathrm{y} ; 8,1 \pm 0,1$ para los conjuntos $\mathrm{SM}, \mathrm{PM}, \mathrm{RU}$ y $\mathrm{SH}$, respectivamente. 


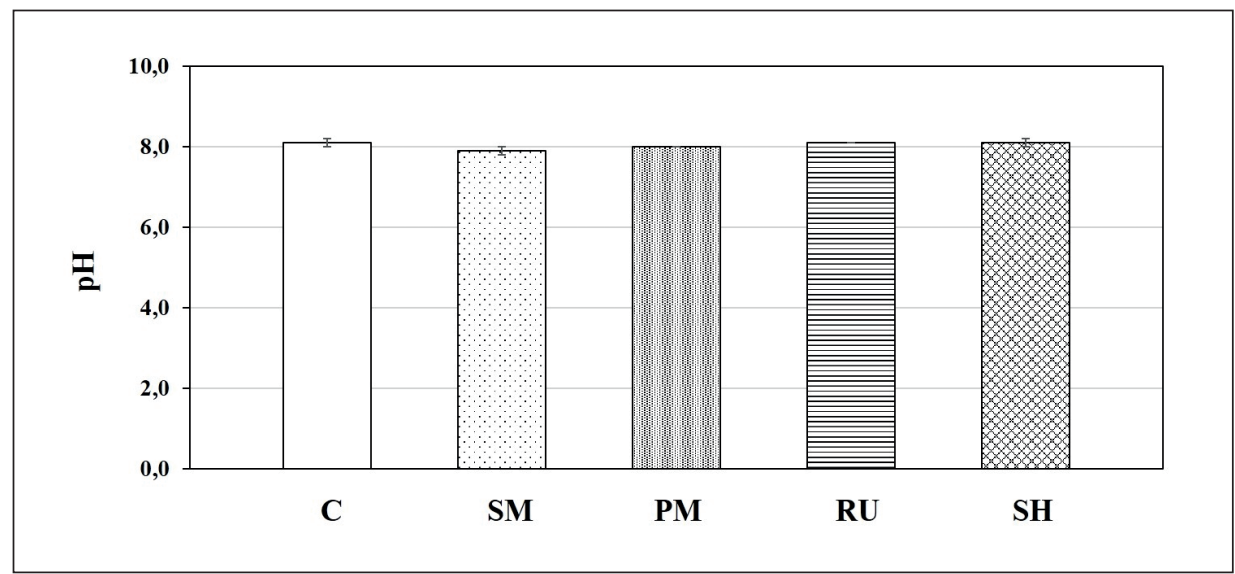

Figura 2. Porcentaje de carbono orgánico del suelo (SOC) seis años después de la repoblación forestal. Dónde: C, suelos reforestados y no enmendados (control); SM, suelos reforestados y enmendados con un acolchado de restos paja $\left(10 \mathrm{Mg} \mathrm{ha}^{-1}\right) ; P M$, suelos reforestados y enmendados con un acolchados de astillas de pino (Pinus halepensis Mill.) (10 $\mathrm{Mg} \mathrm{ha}^{-1}$ ); $R U$, suelos reforestados y enmendados con lodos de depuradora (10 $\left.\mathrm{Mg} \mathrm{ha}^{-1}\right)$; $\mathrm{SH}$, suelos reforestados y enmendados con estiércol de origen vacuno $\left(10 \mathrm{Mg} \mathrm{ha}^{-1}\right)$. * significa diferencias para cada tratamiento respecto al control $(p \leq 0,05)$.

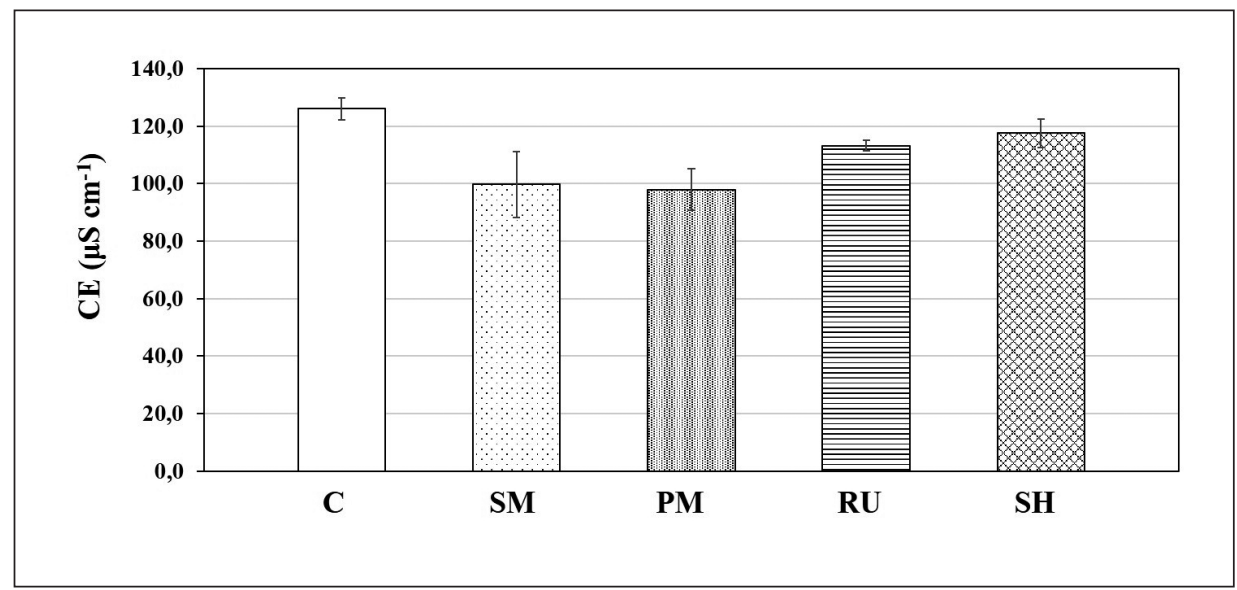

Figura 3. Valores de pH seis años después de la repoblación forestal. Dónde: $C$, suelos reforestados y no enmendados (control); SM, suelos reforestados y enmendados con un acolchado de restos paja (10 $\left.\mathrm{Mg} \mathrm{ha}^{-1}\right)$; PM, suelos reforestados y enmendados con un acolchados de astillas de pino (Pinus halepensis Mill.) (10 $\left.\mathrm{Mg} \mathrm{ha}^{-1}\right) ; R U$, suelos reforestados y enmendados con lodos de depuradora $\left(10 \mathrm{Mg} \mathrm{ha}^{-1}\right)$; SH, suelos reforestados y enmendados con estiércol de origen vacuno $\left(10 \mathrm{Mg} \mathrm{ha}^{-1}\right)$. * significa diferencias para cada tratamiento respecto al control $(p \leq 0,05)$. 
Una tendencia similar a la descrita para el $\mathrm{pH}$, se observa para los valores de $\mathrm{CE}$ (Fig. 4). Los valores de CE fueron muy similares entre manejos y no se observaban diferencias significativas con el control ( $p \geq 0,05$; Fig. 4). Específicamente, los valores de CE medidos en cada tratamiento fueron de $126 \pm 3,9 \mu \mathrm{S} \mathrm{cm}{ }^{-1} ; 99,7 \pm 11,4 \mu \mathrm{S} \mathrm{cm}^{-1}$; $97,9 \pm 7,3 \mu \mathrm{S} \mathrm{cm}^{-1} ; 113,2 \pm 1,74 \mu \mathrm{S} \mathrm{cm}^{-1} \mathrm{y} ; 117,5 \pm 5,0 \mu \mathrm{S} \mathrm{cm}^{-1}$ para los conjuntos $\mathrm{C}, \mathrm{SM}$, PM, RU y SH, respectivamente.

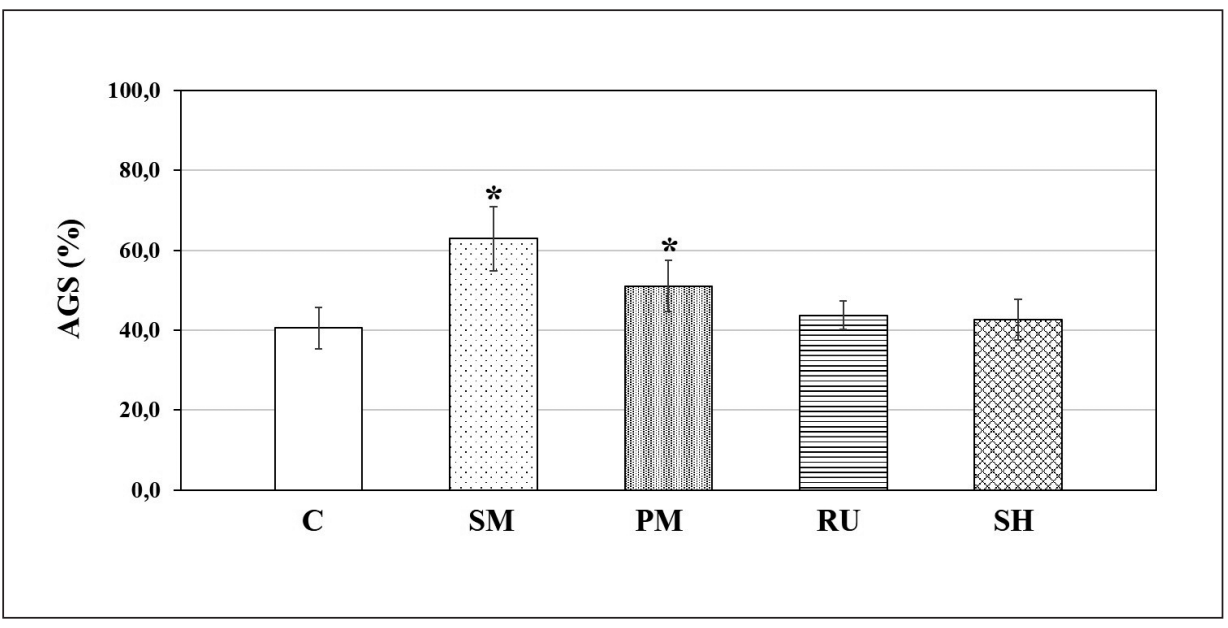

Figura 4. Valores de conductividad Eléctrica $\left(C E, \mu S \mathrm{~cm}^{-1}\right)$ seis años después de la repoblación forestal. Dónde: C, suelos reforestados y no enmendados (control); SM, suelos reforestados y enmendados con un acolchado de restos paja (10 $\left.\mathrm{Mg} \mathrm{ha}^{-1}\right)$; PM, suelos reforestados y enmendados con un acolchados de astillas de pino (Pinus halepensis Mill.) (10 Mg ha ${ }^{-1}$ ); $R U$, suelos reforestados y enmendados con lodos de depuradora (10 $\left.\mathrm{Mg} \mathrm{ha}^{-1}\right)$; $\mathrm{SH}$, suelos reforestados y enmendados con estiércol de origen vacuno $\left(10 \mathrm{Mg} \mathrm{ha}{ }^{-1}\right)$. * significa diferencias para cada tratamiento respecto al control $(p \leq 0,05)$.

\subsection{Propiedades físicas}

La estabilidad estructural media de los agregados (AGS) se representa en la Figura 5. En ella se puede observar un patrón muy similar al descrito para el SOC (Fig. 2). En términos generales, se observa un aumento significativo del AGS en los conjuntos SM y PM ( $p \geq 0,05$; Fig. 5). Así, seis años después de la repoblación forestal, los valores medios de AGS en SM y PM alcanzaron valores de $62,9 \pm 8,1 \%$ y $51,2 \pm 6,4 \%$ respecto a los 40,5 $\pm 5,2 \%$ encontrados en el conjunto C. Por el contrario, en los suelos enmendados con SH y RU, los resultados del ANOVA no mostraban diferencias significativas ( $p \geq 0,05$; Fig. 5), siendo los valores de AGS medidos muy similares a los encontrados en C. De este modo, tras seis años de exposición, los valores de AGS medidos en los conjuntos C, SH y RU alcanzaron valores de 40,5 $\pm 5,2 \% ; 43,7 \pm 3,5 \%$ $\mathrm{y} ; 42,7 \pm 5,1 \%$, respectivamente. 


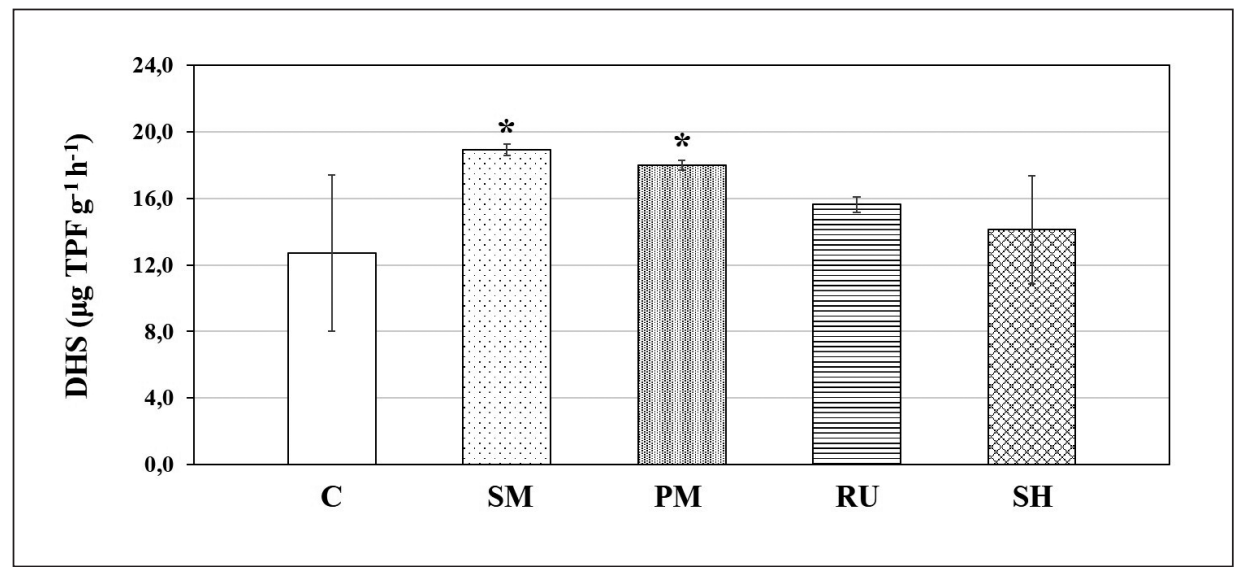

Figura 5. Valores medios de estabilidad estructural de los agregados (AGS, \%) seis años después de la repoblación forestal. Dónde: $C$, suelos reforestados y no enmendados (control); SM, suelos reforestados y enmendados con un acolchado de restos paja (10 $\left.M g \mathrm{ha}^{-1}\right) ; \mathrm{PM}$, suelos reforestados y enmendados con un acolchados de astillas de pino (Pinus halepensis Mill.) (10 $\left.\mathrm{Mg} \mathrm{ha}^{-1}\right) ; R U$, suelos reforestados y enmendados con lodos de depuradora (10 $\left.\mathrm{Mg} \mathrm{ha}^{-1}\right)$; $\mathrm{SH}$, suelos reforestados y enmendados con estiércol de origen vacuno $\left(10 \mathrm{Mg} \mathrm{ha}^{-1}\right)$. significa diferencias para cada tratamiento respecto al control $(p \leq 0,05)$.

\subsection{Propiedades microbiológicas}

En la Figura 6 se encuentran recogidos los valores de la DHS para los cinco manejos. Respecto a la DHS, la tendencia general es encontrar los valores más altos en los dos suelos con acolchados, SM y PM, seguidos sucesivamente de los RU, SH y C (Fig. 6). Sin embargo, aunque los suelos enmendados con RU y SH mostraron valores más altos que los suelos control, el incremento observado no fue significativo ( $p \geq 0,05$; Fig. 6). Por el contrario, en los suelos enmendados con SM y PM, sí se observaron diferencias significativas respecto al control ( $p \leq 0,05$; Fig. 6).

En la Tabla 2 se encuentran recogidos los resultados de las correlaciones lineales entre la DHS y las distintas propiedades físico-químicas del suelo. Se encontró que la DHS correlacionó con todas las variables del suelo, como se puede apreciar en los coeficientes de correlación múltiple y de determinación (Tabla 2). Concretamente, la propiedad que obtuvo un mayor índice de correlación positiva fue el porcentaje de carbono orgánico del suelo (SOC: $\mathrm{r}=0,93$ y $p$-valor $=0,02$ ). Un comportamiento similar se observó al correlacionar la deshidrogenasa (DSH) con el contenido de agregados estables (AGS), donde $\mathrm{r}=0,91 \mathrm{y} p$-valor $=0,03$ (Tabla 2). El pH, por el contrario, presentó una relación opuesta a la encontrada para la DHS respecto al SOC o al porcentaje de AGS ( $r=-0,89$; $p$-valor $=0,05)$. La misma relación inversa se registró entre la enzima DHS y el CE del suelo $(r=-0,98$ significativo a 0,00$)$. 
Tabla 2. Resultados del análisis de regresión múltiple para la actividad de la deshidrogenasa y las propiedades del suelo. Dónde: SOC, carbono orgánico del suelo; AGS, estabilidad estructural media; CE, estabilidad media. Significancia $p \leq 0.05$.

\begin{tabular}{lrrrr}
\hline Estadísticas de la regresión & SOC & AGS & pH & \multicolumn{1}{c}{ CE } \\
\hline Coeficiente de correlación múltiple & 0,93 & 0,91 & $-0,89$ & $-0,98$ \\
Coeficiente de determinación $\mathrm{R}^{2}$ & 0,87 & 0,82 & 0,78 & 0,96 \\
$\mathrm{R}^{2}$ ajustado & 0,82 & 0,76 & 0,71 & 0,95 \\
Error típico & 1,10 & 1,26 & 1,39 & 0,59 \\
$\mathrm{~F}$ & 19,34 & 13,79 & 10,84 & 73,85 \\
-Valor & 0,02 & 0,03 & 0,05 & 0,00 \\
Observaciones & 40 & 40 & 40 & 40 \\
\hline
\end{tabular}

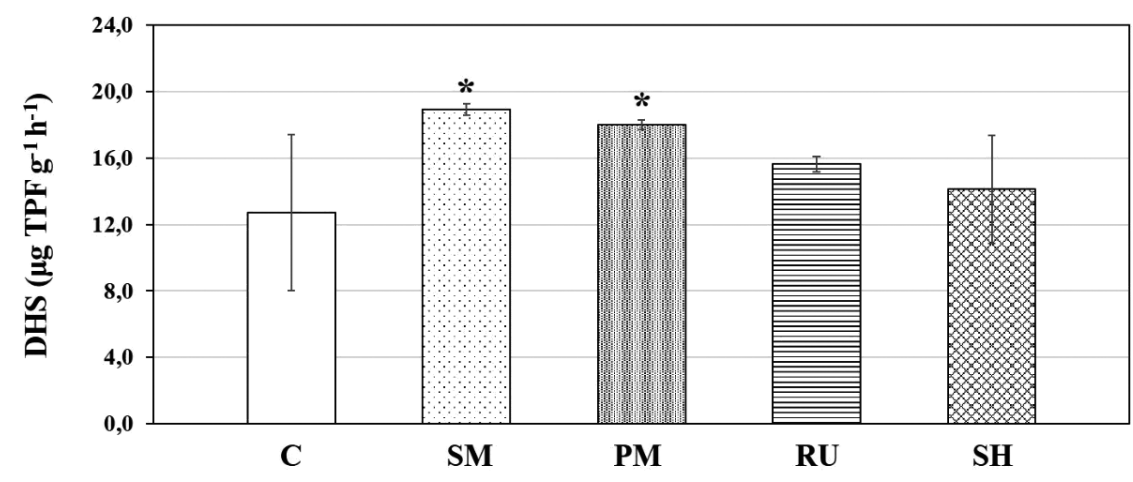

Figura 6. Valores para la enzima deshidrogenasa (DHS, $\left.\mu \mathrm{g} T P F g^{-1} h^{-1}\right)$ seis años después de la repoblación forestal. Dónde: $C$, suelos reforestados y no enmendados (control); SM, suelos reforestados y enmendados con un acolchado de restos paja (10 $\left.\mathrm{Mg} \mathrm{ha}^{-1}\right)$; PM, suelos reforestados y enmendados con un acolchados de astillas de pino (Pinus halepensis Mill.) (10 Mg $\left.h a^{-1}\right) ; R U$, suelos reforestados y enmendados con lodos de depuradora (10 $\left.\mathrm{Mg} \mathrm{ha}^{-1}\right) ; \mathrm{SH}$, suelos reforestados y enmendados con estiércol de origen vacuno $\left(10 \mathrm{Mg} \mathrm{ha}^{-1}\right)$. * significa diferencias para cada tratamiento respecto al control $(p \leq 0,05)$.

\section{Discusión}

\subsection{Cambios estructurales}

El SOC es un importante componente para la restauración de la calidad de los suelos pues está directamente relacionado con la estructura física del mismo, la capacidad de retención de agua y la disponibilidad de nutrientes para las plantas (Brevick, 2009; RuizSinoga et al., 2012; Tesfahunegn, 2013). La materia orgánica de los suelos es el producto de la descomposición química de las excreciones de animales y microorganismos, de 
residuos de plantas o de la degradación de cualquiera de ellos tras su muerte (Eaton et al., 2008; Parras-Alcántara et al., 2013). En este sentido, numerosos estudios han descrito cómo el uso de enmiendas orgánicas podría constituir una manera eficaz de incrementar el SOC y así, a largo plazo, favorecer la estructura y actividad microbiológica de ambientes degradados (Albiach et al., 2001; Roldán et al., 2001; Ferreras et al., 2006). Basándonos en estas premisas, cuando planteamos este estudio, la primera cuestión a resolver fue evaluar qué ocurría con el contenido en carbono orgánico (SOC) seis años después de ejecutar la propuesta forestal. Este estudio muestra que no todas las enmiendas orgánicas resultaron métodos efectivos para incrementar los valores SOC (Fig. 2). Así, seis años después de la adición de las cuatro enmiendas (Fig. 2), únicamente los suelos tratados con acolchados de paja (SM) y poda (PM) habían registrado un incremento significativo en los valores de SOC, respecto a los suelos no enmendados (C). Concretamente, el ratio de incremento medido fue de 2,0 y 1,8, respectivamente (Fig. 2). Los resultados de este estudio sugieren que la adición de enmiendas ayudaba a mantener estable el SOC para los 10 primeros cm del suelo y en los seis primeros años después de la propuesta forestal. De este modo, en érminos generales, el efecto inicial de las enmiendas SM y PM en el suelo fue positivo. Del otro lado, los tratamientos RU y SH no habían resultado efectivos para incrementar de forma significativa los valores de SOC respecto a la condición control $(\mathrm{C})$, mostrando valores muy similares entre ellos ( $\mathrm{SOC}=3,3 \%$ $3,75 \%$ ). Una posible explicación a este hecho fue la baja tasa de mineralización inicial de las enmiendas debido al inexistente grado de compostaje de las enmiendas previo a su aplicación (González-Ubierna et al., 2012; Hueso-González et al., 2014). Este hecho ralentizaría la tasa de mineralización de las mismas en el suelo y sus efectos sobre el SOC tardarían más tiempo en hacerse patentes. En esta misma línea están los resultados de un estudio basado en la velocidad de mineralización de enmiendas con diferentes grados de compostaje (García-Gómez et al., 2005). En él se demostró que en los compost maduros, con mayor grado de compostaje, el contenido de ácidos húmicos era mayor que en los compost inmaduros, lo que aumentaba la tasa mineralización de los mismos una vez aplicados.

El contenido orgánico de un suelo (SOC) se relaciona con la mayoría de los procesos, por no decir con todos, que ocurren en el perfil edáfico (Almendros et al., 2010). En este sentido, los resultados descritos para el SOC están en consonancia con los encontrados para la estabilidad estructural media de los agregados (AGS; Fig. 3). En términos generales, únicamente los tratamientos SM y PM habían conseguido aumentar significativamente los valores medios de AGS respecto a C ( $p \geq 0,05$; Fig.3). Así en ambos tratamientos, el porcentaje de AGS aumentó significativamente en un $22,4 \%$ y $10,7 \%$ frente a la condición de reforestación bajo suelo desnudo (C). Esto se justifica porque el SOC tiene un efecto directo sobre la agregación de las partículas del suelo, ya que actúa como agente cementante de las mismas. Por ello, en los suelos enmendados con PM y SM, el incremento del SOC había contribuido a que las partículas minerales individuales del suelo formasen agregados estables, mejorando así la estructura del suelo. Atendiendo a Mataix-Solera et al. (2010), este hecho implicaría una mayor resistencia a la degradación para los suelos tratados con SM y PM y, por tanto, una mayor protección frente a los procesos de erosión hídrica. La mejora de la estructura edáfica encontrada es 
especialmente relevante en los primeros años post-trasplante, debido a que el tamaño de los brinzales aún es insuficiente para proteger el suelo de la acusada erosión hídrica de ambientes mediterráneos (Arendt, 1997; Benayas, 1998; Erktan et al., 2016). Finalmente, en los suelos enmendados con RU y SH, al igual que lo descrito para el SOC (Fig. 2), la ausencia de diferencias significativas para AGS respecto a $\mathrm{C}(\mathrm{p}>0.05$; Fig. 3$)$ también se hizo manifiesta. Esto conllevaría la presencia de suelos peor estructurados que los encontrados bajo los tratamientos PM y SM y por tanto, más fácilmente erosionables (Ruiz-Sinoga et al., 2009).

\subsection{Cambios en la actividad microbiológica}

Los microorganismos parecen ser los principales agentes para la estabilización de los agregados edáficos (Six et al., 2004; Lehmann et al., 2017). Tanto hongos como bacterias contribuyen a la estabilización de los agregados del suelo a través de la deposición de polisacáridos extracelulares y la formación de materiales húmicos aromáticos degradados que forman los complejos arcilla-metales-materia orgánica (Rillig y Mummey, 2006). Además, juegan un papel directo en la agregación y la estructura del suelo a través de su contribución en los procesos de humificación (Schussler et al., 2001). Así, las bacterias y los hongos exudan polisacáridos coloidales que actúan como pegamento entre las partículas del suelo (Parniske, 2008). Por todo ello, presentan un interesante potencial como indicadores de alta sensibilidad a los cambios edáficos producidos por las prácticas de manejo y pueden ser utilizados como indicadores potenciales de calidad de los suelos (Barto et al., 2010; Hallett et al., 2009; Martin et al., 2012).

En este estudio, la enzima Deshidrogenasa (DHS) se ha utilizado como indicador para evaluar el efecto de las enmiendas orgánicas sobre características de calidad o estado de sanidad del suelo en condiciones semiáridas mediterráneas (Gajda y Mortyniuk, 2005; Baležentiené y Klimas, 2009). Esto es posible porque la actividad de la DHS permite, de manera global, tener una idea de los procesos microbianos que ocurren en el suelo, indicando además la tasa de oxidación de la materia orgánica de las enzimas involucradas en el ciclo del C (Kuhur et al., 2012). En este estudio, la DHS mostró diferencias estadísticamente significativas dependientes del tipo de tratamiento aplicado al suelo ( $p \leq 0,05$; Fig. 6). Al igual que lo descrito para AGS y SOC (Figs. 2 y 3), la tendencia general es a encontrar los valores de DHS más altos en los suelos tratados con acolchados orgánicos, SM y PM (Fig. 6). Los resultados obtenidos indican claramente que la actividad microbiológica refleja la existencia del gradiente de intensidad según el manejo del suelo estudiados, $\mathrm{SM}>\mathrm{PM}>\mathrm{RU}>\mathrm{SH}>\mathrm{C}$. Sin embargo, cabe decir que no se observan diferencias significativas para la DHS para los tratamientos RU y SH respecto a $\mathrm{C}(\mathrm{p} \geq 0.05$; Fig. 6$)$. El incremento significativo de la actividad para la DHS, que si fue observado en los tratamientos SM y PM, es atribuido al aporte extra de SOC anteriormente descrito (Fig. 2). Así, se evidencia también en los coeficientes de correlación y determinación encontrados en la tabla 2. Concretamente, se observó al correlacionar la deshidrogenasa (DSH) con el contenido AGS o de SOC una correlación positiva muy alta $\left(r>0,90 ; R^{2}>0,80\right)$, lo que indicaba que el aumento del SOC podría ser la causa principal del incremento de la actividad microbiana del suelo (Marcote et 
al., 2001). Esto es posible porque la actividad de la DHS del suelo refleja la actividad total oxidativa de los microorganismos sobre la materia orgánica (Rillig y Mummey, 2006). Siendo el resultado global de las diferentes deshidrogenasas que participan en el metabolismo respiratorio, en el ciclo del citrato y en el metabolismo del nitrógeno (Trasar et al., 2003). Las deshidrogenasas son enzimas exclusivamente intracelulares que únicamente están presentes en las células vivas (Kuhur et al., 2012). Al no acumularse extracelularmente en el suelo, su media refleja la cantidad de microorganismos viables y su capacidad oxidativa en el momento de la determinación (Schussler et al., 2001; Lehmann et al., 2017). Los resultados obtenidos en este trabajo apoyan esta conclusión. Podemos afirmar, por tanto, que la adición de enmiendas orgánicas de tipo acolchado de paja (SM) o poda (PM) había supuesto un incremento de los valores de SOC respecto al C (Fig. 2). Además, este aumento estaba directamente relacionado el incremento de la actividad microbiológica, tal como muestran los valores de la DHS en la figura 5 (Juarez et al., 2013). Esto podría haber acelerado los procesos de humificación de la materia orgánica, implicando un aumento del número de agentes cementantes de las partículas minerales, lo que justifica el incremento de la estabilidad de los agregados (Fig. 5) (Yazdanpanah et al., 2016). Por tanto, desde el punto de vista de la gestión forestal el uso de acolchados de paja (SM) y poda (PM) resultan métodos altamente efectivos para mejorar la estructura del suelo en los primeros años después de la reforestación y donde la vegetación aún no tiene el porte suficiente para proteger al suelo frente a los acusados fenómenos de erosión hídrica que afectan a las áreas mediterráneas (Hueso-González et al., 2017).

\subsection{Cambios en la acidez y salinidad}

En un suelo forestal, la salinidad influye directamente en la viabilidad y crecimiento del plantón (Allakherdiez et al., 2000; Parida y Das, 2005; MorugánCoronado et al., 2011). En este sentido, hay autores que afirman que la adición de enmiendas orgánicas al suelo, podría suponer una alteración de los valores naturales de salinidad (Casado-Vela et al., 2007; Li et al., 2007; Shazana et al., 2013; Srinivasarao et al., 2013). Esto mismo evidencian las correlaciones encontradas en la tabla 2 (r $=-0,92$ ), donde se puede observar una correlación alta y negativa entre la actividad enzimática de la DHS y el incremento de la CE. Por ello, ante los tratamientos de fertilización orgánica es sumamente importante tener en consideración cualquier posible variación de la $\mathrm{CE}$, pues una alteración de la salinidad del suelo podría conllevar la aparición de efectos secundarios que interfieran de modo negativo en el desarrollo de la macro o microflora edáfica (Gajda y Mortyniuk, 2005; Bastidia et al., 2007). Así lo habían demostrado también Allakherdiez et al. (2000) y Parida y Das (2005). Según estos autores, en un suelo forestal al que se alteró la salinidad mediante la adición de lodos de depuradora, se observaban grandes alteraciones tanto en la microbiota edáfica como el desarrollo de los plantones (Allakherdiez et al., 2000; Parida y Das, 2005). Sin embargo, cuando atendíamos a los resultados de nuestro estudio, cabe decir, que en ninguno de los tratamientos se observaron diferencias significativas en la CE con respecto a los suelos control (C) (Fig. 4). Así, con independencia del tratamiento aplicado, los valores de CE medidos fueron 
siempre similares a la condición natural (Tabla 1) e inferiores a $2.000 \mu \mathrm{S} \mathrm{cm}^{-1}$, que es el límite establecido por Marañes et al. (1994) para clasificar un suelo como salino y para que puedan darse afecciones serias en el desarrollo de la vegetación.

Al igual que la salinidad, la acidez del suelo influye directamente en la supervivencia de los individuos reforestados (Parida y Das, 2005). Así se evidencia en la Tabla 2 ( $\mathrm{r}=$ $-0,89)$. De este modo, el pH afecta específicamente a la disponibilidad de los nutrientes de las plantas, mediante el control de las formas químicas de los mismos (Allakherdiez et al., 2000). Existen estudios que demostraron que el $\mathrm{pH}$ del suelo podía variar por la adición de enmiendas al mismo (Guang-Ming et al., 2006). Estos estudios, además, demostraron que las variaciones introducidas eran dependientes de la cantidad y tipo de enmienda utilizada (González-Fernández et al., 2003). Así, Méndez et al. (2012) y Bernal et al. (1992) indicaron diferencias significativas respecto a los suelos no enmendados, tras la aplicación de algunas enmiendas orgánicas a suelos agrícolas. En contraposición, nuestros resultados reflejan que pese a que los tratamientos habían modificado ligeramente el $\mathrm{pH}$ respecto a los suelos control. Sin embargo, no se pueden considerar las variaciones encontradas significativas como para poder determinar el efecto directo de cada enmienda en el $\mathrm{pH}$ o para que la supervivencia de la vegetación se viese afectada ( $p>0,05$; Fig. 3 ).

\section{Conclusiones}

Tras la adición de cuatro enmiendas orgánicas como complemento a un plan de repoblación forestal y bajo condiciones secas mediterráneas, se observan dos tendencias totalmente opuestas: (i) enmiendas que resultan efectivas para favorecer los procesos de estructuración del suelo; y (ii) enmiendas que no resultan efectivas para favorecer los procesos de estructuración del suelo. En este sentido, seis años después de su aplicación, únicamente la adición de enmiendas orgánicas, tipo acolchado de paja o poda, había supuesto un incremento significativo de los valores de SOC respecto a la reforestación bajo condiciones de suelo desnudo. Este aumento estaba directamente relacionado con el aumento de la actividad microbiológica, implicando a su vez el incremento del número de agentes cementantes para la unión de las partículas minerales del suelo, lo que justificaba el aumento de la estabilidad media de los agregados encontrado. Además, los acolchados habían resultado inocuos para la salinidad y acidez del suelo, por lo que su uso no conllevaría efectos nocivos para el desarrollo de los plantones. Por tanto, desde el punto de vista de la gestión forestal el uso de acolchados de paja y poda podrían resultar métodos altamente efectivos para mejorar la estructura del suelo y favorecer la microbiota edáfica. Esta mejora de la estructura es especialmente relevante en los primeros años post-transplante, ya que la vegetación aún no tiene el porte suficiente para proteger al suelo frente a los acusados fenómenos de erosión hídrica que afectan a las áreas mediterráneas. Del otro lado, en los tratamientos lodos de depuradora o estiércol de origen vacuno, la ausencia de diferencias significativas en todas las propiedades estudiadas respecto al control pone de manifiesto que, bajo las condiciones de este estudio, dichos tratamientos no resultan 
métodos efectivos para favorecer la estructura edáfica y, por tanto, no serían métodos útiles de gestión frente a la condición de suelo desnudo.

\section{Agradecimientos}

Este estudio ha sido financiado por el proyecto de investigación P09-RNM-5057 (Consejería de Innovación, Ciencia y Empleo de la Junta de Andalucía), así como por el Campus de Excelencia Internacional de Andalucía Tech. Finalmente, los autores quieren agradecer a la empresa TRAGSA su apoyo técnico durante la puesta en marcha de la red experimental.

\section{Referencias}

Albiach, R., Canet, R., Pomares, F., Ingelmo, F. 2001. Organic matter components, aggregate stability and biological activity in a horticultural soil fertilized with different rates of two sewage sludges during ten years. Bioresource Technology 77, 109-114. https://doi. org/10.1016/S0960-8524(00)00166-8.

Allakherdiez, S.I., Sakamoto, A., Nishiyama, Y., Murata, N. 2000. Ionic and Osmotic Effects of $\mathrm{NaCL}-$ Induced Inactivation of Photosystems and I and II in Synechococcus sp. American Society of Plant Physiologists 123, 1047-1056. https://doi.org/10.1104/pp.123.3.1047.

Almendros, G., Gonzalez-Vila, J., González-Pérez, J.A., Knicker, H., de la Rosa, J.M. 2010. Protocolos y técnicas para evaluar el impacto del fuego sobre la materia orgánica. En: A. Cerdá, A. Jordán (Eds.), Actualización en métodos y técnicas para el estudio de los suelos afectados por incendios forestales. Valencia, Cátedra de Divulgación de la Ciencia, pp. 291319.

Arendt, J.D. 1997. Adaptive intrinsic growth rates: an interpretation across taxa. Quarterly Review of Biology 72, 149-177. https://doi.org/10.1086/419764.

Baležentienè, L., Klimas, E. 2009. Effect of organic and mineral fertilizers and land management on soil enzyme activities. Agronomy Research (Special issue I) 7, 191-197.

Barto, E.K., Alt, F., Oelmann, Y., Wilcke, W., Rillig, M.C. 2010. Contributions of biotic and abiotic factors to soil aggregation across a land use gradient. Soil Biology and Biochemistry 42, 2316-2324. https://doi.org/10.1016/j.soilbio.2010.09.008.

Benayas, L.M.1998. Drougth and survival Quercus ilex L. seedlings after irrigation and artificial shading on Mediterranean set-aside agricultural land. Annales des Sciences Forèstieres 55, 801-807. https://doi.org/10.1051/forest:19980704.

Bernal, M.P., Roig, A., Lax, A., Navarro, A.F. 1992. Effects of the application of pig slurry on some physico-chemical and physical properties of calcareous soils. Bioresource Technology 42, 233-239. https://doi.org/10.1016/0960-8524(92)90026-T.

Bretón, V., Crosaz, Y., Rey, F. 2016. Effect of wood chip amendments on the revegetation performance of plant species on eroded marly terrains in a Mediterranean mountainous climate (Southern Alps, Alpes). Solid Earth 7, 599-610. https://doi.org/10.5194/se-7-5992016.

Brevick, E.C. 2009. Soil health and productivity. En: W. Verheye (Ed.), Soils, plant growth and crop production. Encyclopedia of Life Support Systems (EOLSS), EOLSS Publishers, Oxford, UK. http://www.eolss.net. (accessed 5/27/2014).

Casado-Vela, J., Sellés, S., Díaz-Crespo, C., Navarro-Pedreño, J., Mataix- Beneyto, J., Gómez, I. 2007. Effect of composted sewage sludge application to soil on sweet pepper crop (Capsicum 
annuum varannuиm) grown under two exploitation regimes. Waste Management 27, 15091518. https://doi.org/10.1016/j.wasman.2006.07.016.

Casals, P., Cortina, J., Bottner, P., Coûteaux, M.M., Vallejo, V.R. 2000. CO2 efflux from a Mediterranean semi-arid forest soil. I. Seasonality and effects of stoniness. Biogeochemistry 48, 261-281. https://doi.org/10.1023/A:1006289905991.

Casida, L., Klein, D., Santoro, T. 1964. Soil dehydrogenase activity. Soil Science 98, 371-376. https://doi.org/10.1097/00010694-196412000-00004.

COM 2002. Towards a thematic strategy for soil production. Communication for the Commission to the Council, the European Parliament, the Economic and Social Committee and the Committee and the Committee of the Regions. COM (2002). 179 end. Brussels, 16.4.2002.

DMR 2008. Directiva 2008/98/CE del parlamento europeo y del consejo de 19 de noviembre de 2008 sobre residuos. Diario oficial de la unión europea. 312/3 pp.

Dudás, P., Gedeon, C., Menyhárt, L., Ambrus, G., Tóth, F. 2016. The effect of mulching on the abundance and diversity of ground beetle assemblages in two hungarian potato fields. Journal of Agricultural and Environmental Sciences 3, 45-53. https://doi.org/10.18380/ SZIE.COLUM.2016.3.1.45.

Eaton, J.M., McGoff, N.M., Byrne, K.A., Leahy, P., Kiely, G. 2008. Land cover change and soil organic C stocks in the Republic of Ireland 1851-2000. Climatic Change 91,317-334. https:// doi.org/10.1007/s10584-008-9412-2.

Erkant, A., Cécillon, L., Graf, F., Roumet, C., Legout, C., Rey, F. 2016. Increase in soil aggregate stability along Mediterranean successional gradient in severely eroded gully bed ecosystems: combined effects of soil, root traits and plants community characteristics. Plant and Soil 398, 121-137. https://doi.org/10.1007/s11104-015-2647-6.

FAO 2006. World reference base for soil resource. A framework for a international classification, correlation and communication. FAO, Roma, $145 \mathrm{pp}$.

Ferreras, L., Gómez, E., Torresani, S., Firpo, I., Rotondo, R. 2006. Effect of organic amendments on some physical, chemical and biological properties in a horticultural soil. Bioresource Technology 97, 635-640. https://doi.org/10.1016/j.biortech.2005.03.018.

Flanagan, D.C., Norton, L.D., Shainberg, I. 1997a. Effect of water chemistry and soil amendments on a silt loam soil-Part 1: infiltration and runoff. Transactions of the ASAE 46 (6), 1549-1555. https://doi.org/10.13031/2013.21419.

Flanagan, D.C., Norton, L.D., Shainberg, I. 1997b. Effect of water chemistry and soil amendments on a silt loam soil-Part 2: soil erosion. Transactions of the ASAE 46 (6), 1555-1561.

Gajda, A., Martyniuk, S. 2005. Microbial Biomass C and N and Activity of Enzymes in Soil under Winter Wheat Grown in Different Crop Management Systems. Polish Journal of Environmental Studies 14, 159-163.

García-Gómez, A., Bernal-Calderón, M.P., Roig, A. 2005. Humification parameters, organic waste management, organic matter. Compost Science and Utilization 13, 127-135. https://doi.org/1 0.1080/1065657X.2005.10702229.

Gholami, L., Reza Sadeghi, S.H., Homaee, M. 2012. Efficiency of rice straw mulch as a soil amendment to reduce splash erosion. Erosion and Sediment Yields in the Changing Environment. IAHS Publication 356, 11-15.

González-Fernández, P., Ordóñez-Fernández, R., Espejo-Serrano, R., Peregrini-Alonso, F. 2003. Cambios del pH del perfil de un suelo acido cultivado y enmendado con diversos materiales para incrementar su fertilidad. En: P. Álvarez-Benedí, P. Marinero (Eds), Estudios de la zona no saturada del suelo, Vol. VI.

González-Ubierna, S., Jorge-Mardomingo, I., Carrero-González, B., De la Cruz, M.T., Carsermeiro, M.A. 2012. Soil organic matter evolution after the application of high doses of organic 
amendments in a Mediterranean calcareous soil. Soils and Sediments 12, 1257-1268. https:// doi.org/10.1007/s11368-012-0516-y.

Guang-Ming, L., Jing-Song, Y., Rong-Jiang, Y. 2006. Electrical conductivity in soil extracts: chemical factors and their intensity. Pedosphere 16, 100-107.

Guerrero, C., Gómez, I., Moral, R., Mataix-Solera, J., Mataix-Beneyto, J., Hernández, T. 2001. Reclamation of a burned forest soil with municipal waste compost: macronutrient dynamic and improved vegetation cover recovery. Bioresource Technology 76, 221-227. https://doi. org/10.1016/S0960-8524(00)00125-5.

Hallett, P.D., Feeney, D.S., Bengough, A.G, Rillig, M.C., Scrimgeour, C.M., Young, I.M. 2009. Disentangling the impact of AM fungi versus roots on soil structure and water transport. Plant Soil 314, 183-196. https://doi.org/ https://doi.org/10.1007/s11104-008-9717-y.

Hueso-González, P., Martínez-Murillo, J.F., Ruiz Sinoga, J.D. 2014. The impact of organic amendments on forest soil properties under Mediterranean climatic conditions. Land Degradation \& Development 25, 604-612. https://doi.org/10.1002/ldr.2296.

Hueso-González, P., Martínez-Murillo, J.F., Ruiz Sinoga, J.D. 2016. Effects of topsoil treatments on afforestation in a dry-Mediterranean climate (Southern Spain). Solid Earth 7, 1479-1489. https://doi.org/10.5194/se-2016-98.

Hueso-González, P., Martínez-Murillo, J.F., Ruiz Sinoga, J.D. 2017. Beneficios de los acolchados de paja y poda como prácticas para la gestión forestal de montes mediterráneos. Cuadernos de Investigación Geográfica 43 (1), 189-208. https://doi.org/10.18172/cig.3142.

Hueso-González, P., Martínez-Murillo, J.F., Ruiz Sinoga, J.D. 2018. Benefits of adding forestry clearance residues for the soil and vegetation of a Mediterranean mountain forest. Science of The Total Environment 615, 796-804. https://doi.org/10.1016/j.scitotenv.2017.09.301.

Hueso-González, P., Ruiz Sinoga, J.D., Martínez-Murillo, J.F., Lavee, H. 2015. Overland flow generation mechanisms affected by topsoil treatment: Application to soil conservation. Geomorphology 228, 796-804. https://doi.org/10.1016/j.geomorph.2014.10.033.

Iglesias, J.O., Miglierina, A.M., Minoldo, G.V., Rodrriguez, R.A., Ayastuy, M.E. 2015. Efecto de la aplicación de diferentes enmiendas orgánicas sobre algunas propiedades edáficas. En: E. de Sá Pereira, G. Minoldo, J.A. Galantini (Eds.), Impacto de los sistemas actuales de cultivo sobre las propiedades químicas del suelo. Efectos sobre los balances de carbono. Ediciones INTA, Buenos Aires.

ISRIC. 2002. Procedures for soil analysis. Internacional Soil Reference and Information Center, Food and Agriculture Organization of the United Nations, Wageningen, The Netherlands.

Jiménez, M.N., Fernández-Ondoño, E., Ripoll, M.A., Castro-Rodríguez, J., Huntsinger, L., Bruno Navarro, F. 2013. Stones and organic mulches improve the Quercus Ilex L. afforestation success under Mediterranean climatic conditions. Land Degradation \& Development 27, 357-365. https://doi.org/10.1002/ldr2250.

Jordán, A., Zavala, L.M., Gil, J. 2010. Effects of mulching on soil physical properties and runoff under semi-arid conditions in southern Spain. Catena 81, 77-85. https://doi.org/10.1016/j. catena.2010.01.007

Jordán, A., Zavala, L.M., Muñoz-Rojas, M. 2011. Mulching, effects on soil physical properties. En: J. Glinski, J. Horabik, J. Lipiec (Eds.), Encyclopedia of Agrophysics. Springer, Berlin, pp. 492-496. https://doi.org/10.1007/978-90-481-3585-1_275.

Juarez, S., Nunan, N., Duday, A.-C., Pouteau, V., Chenu, C. 2013. Soil carbon mineralisation responses to alterations of microbial diversity and soil structure. Biology and Fertility of Soils 49, 939-948. https://doi.org/10.1007/s00374-013-0784-8.

Keesstra, S.D., Bouma, J., Wallinga, J., Tittonell, P., Smith, P., Cerdà, A., Montanarella, L., Quinton, J.N., Pachepsky, Y., Van der Putten, W.H., Bardgett, R. D., Moolenaar, S., Mol, G., Jansen, B., Fresco, L.O. 2016. The significance of soils and soil science towards realization of 
the United Nations sustainable development goals. SOIL 2, 111-128. https://doi.org/10.5194/ soil-2-111-2016.

Keesstra, S., Nunes, J., Novara, A., Finger, D., Avelar, D., Kalantari, Z., Cerdà, A. (2018). The superior effect of nature based solutions in land management for enhancing ecosystem services. Science of the Total Environment 610, 997-1009. https://doi.org/10.1016/j. scitotenv.2017.08.077.

Kemper, M.D., Rosenau, R.C. 1986. Aggregate stability and particle size distribution. En: A. Klute (Ed.), Methods of Soil Analysis, Part 1. American Society of Agronomy. Madison, Wisconsin, pp. 456-442.

Kuhur, M., Gartia, S.K., Patel, A.K. 2012. Quantifying the contribution of diferent soil properties on enzyme activities in dry tropical ecosystems. Journal of Agricultural and Biological Science 7 (9), 763-773.

Lavee, H., Imeson, A.C., Pariente, S. 1998. The impact of climate change on geomorphology and desertification along a Mediterranean arid transect. Land Degradation \& Development 9, $407-$ 422. https://doi.org/10.1002/(SICI)1099-145X(199809/10)9:5<407::AID-LDR302>3.0.Co;2-6.

Lehmann, A., Leifheit, E.F., Rillig, M.C. 2017. Mycorrhizas and Soil Aggregation. Mycorrhizal Mediation of Soil. Fertility, Structure, and Carbon Storage 14, 2041-262.

Li, X., Li, Z., Ding, Y., Liu, S., Zhao, Z., Luo, L., Pang, H., Li, C., Li, H., You, X., Wang, F. 2007. Seasonal variations of $\mathrm{PH}$ and electrical conductivity in a snow-firn pack on Glacier No, 1, eastern Tianshan, China. Cold Regions Science and Technology 48, 55-63. https://doi. org/10.1016/j.coldregions.2006.09.006.

LUCDEME. 1986. Proyecto LUCDEME. Mapa de suelos 1:100.000. Motril-1055. Ministerio de Agricultura, Pesca y Alimentación. ICONA. J. Aguilar (Ed.). Universidad de Granada. Facultad de Ciencia. Departamento de Edafología.

Luna, L., Miralles, I., Andrenelli, M.V., Gispert, M., Pellegrini, S., Vignozzi, N., Solé-Benet, A. 2016. Restoration techniques affect soil organic carbon, glomalin and aggregate stability in degraded soils of a semiarid Mediterranean region. Catena 143, 256-264. https://doi. org/10.1016/j.catena.2016.04.013.

Maestre, F.T., Cortina, J., Bautista, S., Bellot, J., Vallejo, V.R. 2003. Small-scale environmental heterogeneity and spatial-temporal dynamics of seedling establishment in a semiarid degraded ecosystem. Ecosystems 6, 630-643. https://doi.org/10.1007/s10021-002-0222-5.

Madari, B., Machado, P., Torres, E., De Andagre, A.G., Valencia, L. 2005. No tillage and crop rotation effects on soil aggregation and organic carbon in a Rhodic Ferrasol from Surthern of Brazil. Soil and Tillage Research 82, 185-200. https://doi.org/10.1016/j.still.2004.03.006.

Marañes, A., Sánchez Garrido, J.A., De Haro Lozaño, S., Sánchez Gómez, S.T., Lozano Cantarero, F.J. 1994. Análisis de suelo, metodología e interpretación. Servicio de Publicaciones de la Universidad de Almería, Almería.

Marcote, I., Hernández, T., García, C., Polo, A. 2001. Influence of one or two successive annual applications of organic fertilizers on the enzyme activity of a soil under barley cultivation. Biorecourse Technology 79,147-154. https://doi.org/10.1016/S0960-8524(01)00048-7.

Martin, S.L., Mooney, S.J., Dickinson, M.J., West, H.M. 2012. Soil structural responses to alterations in soil microbiota induced by the dilution method and mycorrhizal fungal inoculation. Pedobiologia 55, 271-281. https://doi.org/10.1016/j.pedobi.2012.06.001.

Martínez-Murillo, J.F., Hueso-González, P., Ruiz-Sinoga, J.D. 2017a. Impact of low pressure grazing in the hydrological and sediment connectivity in hillslopes under contrasted Mediterranean climatic conditions (South of Spain). Land Degradation \& Development. https://doi.org/10.1002/ldr.2809. 
Martínez-Murillo, J.F., Hueso-González, P., Ruiz-Sinoga, J.D. 2017b. Topsoil moisture mapping using geostatistical techniques under different Mediterranean climatic conditions. Science of The Total Environment 595, 400-412. https://doi.org/10.1016/j.scitotenv.2017.03.291.

Martínez-Murillo, J.F., Hueso-González, P., Ruiz-Sinoga, J.D.,Lavee,H. 2016. Short-Experimental fire effects in soil and water losses in southern of Spain. Land Degradation \& Development 27, 1513-1522. https://doi.org/10.1002/ldr.2504.

Mataix-Solera, J., Benito, E., Andreu, V., Cerdá, A., Llovet, K., Úbeda, X., Martí, C., Varela, E., Gimeno, E., Arcenegu, V., Rubio, J.L., Campo, J., García-Orenes, F., Badía, D. 2010. ¿Cómo estudiar la estabilidad de agregados en suelos afectados por incendios? Métodos de interpretación de resultados. En: A. Cerdá, A. Jordán (Eds), Actualización de métodos y técnicas para el estudio de los suelos afectados por incendios forestales. FUEGORED, Valencia, Cátedra de Divulgación de la Ciencia, pp. 113-131.

Méndez, A., Gómez, A., Paz-Ferreiro, J., Gascó, G. 2012. Effects of sewage sludge biochar on plant metal availability after application to a Mediterranean soil. Chemosphere 89, 13541359. https://doi.org/10.1016/j.chemosphere.2012.05.092.

Morugán-Coronado, A., García-Orenes, F., Mataix-Solera, J., Arcenegui, V., Mataix-Beneyto, J. 2011. Short-term effects of treated wastewater irrigation on Mediterranean calcareous soil. Soil and Tillage Research 112, 18-26. https://doi.org/10.1016/j.still.2010.11.004.

Muñoz-Rojas, M., Erickson, T.E., Dixon, K.W., Merritt, D. 2016. Soil quality indicators to assess functionality of restored soils in degraded semiarid ecosystems. Restoration Ecology 24, 4352. https://doi.org/10.1111/rec.12368.

Nadal-Romero, E., Cammeraat, E., Pérez-Cardiel, E., Lasanta, T. 2016. Effect of secondary succession and afforestation practices on soil properties after cropland abandonment in humid Mediterranean mountain areas. Agriculture, Ecosystems and Environment 228, 91100. https://doi.org/10.1016/j.agee.2016.05.003.

Ojeda, G., Alcañiz, J.M., Ortiz, O. 2003. Runoff and losses by erosion in soils amended with sewage sludge, Land Degradation \& Development 14 (6), 563-573. https://doi.org/10.1002/ ldr.580.

Ojeda, G., Ortiz, O., Medina, C.R., Perera, J.M. 2015. Carbon sequestration in a limestone quarry mine soil amended with sewage sludge. Soil Use and Management 31, 270-278. https://doi. org/10.1111/sum.12179.

Pal, P.K., Mahajan, M. 2017. Tillage system and organic mulch influence leaf biomass, steviol glycoside yield and soil health under sub-temperate conditions. Industrial Crop and Products 104, 33-44. https://doi.org/10.1016/j.indcrop.2017.04.012.

Parida, A.K., Das, A.B. 2005. Salt tolerance and salinity effects on plants: a review. Ecotoxicology and Environmental Safety 60, 324-349. https://doi.org/10.1016/j.ecoenv.2004.06.010.

Parras-Alcántara, L., Díaz-Jaimes, L., Lozano-García, B. 2013. Organic farming affects C and N in soils under olive groves in Mediterranean areas. Land Degradation \& Development 26 (8), 800-806. https://doi.org/10.1002/ldr.2231.

Parniske, M. 2008. Arbuscular mycorrhiza: the mother of plant root endosymbiosis Nature Reviews Microbiology 6, 763-775. https://doi.org/10.1038/nrmicro1987.

Prats, S.A., Dos Santos Martins, A., Cortizo Malvar, M., Ben-Hur, M., Keizer, J.J. 2014. Polyacrylamide application versus forest residue mulching for reducing post-fire runoff and soil erosion. Science of Total Environment 468-469, 464-474. https://doi.org/10.1016/j. scitotenv.2013.08.066.

Prosdocimi, M., Jordán, A., Tarolli, P., Keesstra, S., Novara, A., Cerdà, A. 2016. The immediate effectiveness of barley straw mulch in reducing soil erodibility and surface runoff generation in Mediterranean vineyards. Science of the Total Environment 547, 323-330. https://doi. org/10.1016/j.scitotenv.2015.12.076. 
Rillig, M.C., Mummey, D.L. 2006. Mycorrhizas and soil structure. New Phytologist 171, 41-53. https://doi.org/10.1111/j.1469-8137.2006.01759.x.

Roldán, A., Albaladejo, J., Thormes, J.B. 2001. Aggregate stability changes in a semiarid soil after treatment with different organic amendments. Bioresource Technology 76, 125-129. https:// doi.org/10.1016/S0960-8524(00)00090-0.

Ruiz-Sinoga, J.D., Martínez Murillo, J.F. 2009. Eco-geomorphological system response variability to the 2004-06 drought along a climatic gradient of the Littoral Betic Range (southern Spain). Geomorphology 103, 351-362. https://doi.org/10.1016/j.geomorph.2008.07.002.

Ruiz-Sinoga, J.D., Pariente, S., Romero Díaz, A., Martínez Murillo, J.F. 2012. Variability of relationships between soil organic carbon and some soil properties in Mediterranean rangelands under different climatic conditions (South of Spain). Catena 94, 17-25. https:// doi.org/10.1016/j.catena.2011.06.004.

Schussler, A., Schwarzott, D., Walker, C. 2001. A new fungal phylum, the Glomeromycota: phylogeny and evolution. Mycological Research 105, 1413-1421. https://doi.org/10.1017/ S0953756201005196.

Shazana, M.A.R.S., Shamshuddin, J., Fauziah, C.I., Syed-Omar, S.R. 2013. Alleviating the infertility of an acid sulphate soil by using ground basalt with or without lime and organic fertilizer under submerged conditions. Land Degradation \& Development 24, 129-140. https://doi.org/10.1002/ldr.11111.

Six, J., Bossuyt, H., Degryze, S., Denef, K. 2004. A history of research on the link between (micro) aggregates, soil biota, and soil organic matter dynamics. Soil and Tillage Research 79 (1), 7-31. https://doi.org/10.1016/j.still.2004.03.008.

Smets, T., Poesen, J., Bochet, E. 2008. Impact of plot length on the effectiveness of different soilsurface covers in reducing runoff and soil loss by water. Progress in Physical Geography 32 (6), 654-677. https://doi.org/10.1177/0309133308101473.

Srinivasarao, C.H., Venkateswarlu, B., Lal, R., Singh, A.K., Kundu, S., Vittal, K.P.R., Patel, J.J., Patel, M.M. 2013. Long-term manuring and fertilizer effects on depletion of soil organic carbon stocks under pearl millet-cluster bean-castor rotation in Western India. Land Degradation \& Development 25, 173-183. https://doi.org/10.1002/ldr.1158.

Tejada, M., González, J.L. 2003. Effects of the application of a compost originating from crushed cotton gin residues on wheat yield under dryland conditions. European Journal of Agronomy 19 (2), 357-368. https://doi.org/10.1016/S1161-0301(02)00089-8.

Tesfahunegn, G.B. 2013. Soil quality indicators response to land use and soil management systems in Northern Ethiopia's Catchment. Land Degradation \& Development 27 (2), 438-448. https://doi.org/10.1002/ldr.2245.

Trasar, C., Leiros, M., Gil, F. 2003. Consideraciones generales sobre la determinación de las actividades enzimáticas del suelo. En: C. García, F. Gil, T. Hernández, C. Trasar (Eds.), Técnicas de análisis de parámetros bioquímicos en suelos. Mundiprensa, Madrid.

Wahbi, A., Avery, A., Dercon, G., Heng, L. 2017. Mitigating Soil Moisture Evaporation via Organic Mulch Application in Cultivated Agricultural Environments (INIS-XA--17M0675). International Atomic Energy Agency (IAEA): IAEA.

Yazdanpanah, N., Mahmoodabadi, M., Cerdà, A. 2016. The impact of organic amendments on soil hydrology, structure and microbial respiration in semiarid lands. Geoderma 266, 58-65. https://doi.org/10.1016/j.geoderma.2015.11.032. 